$\xi=$ 줄

\title{
Pleomorphic adenoma of the upper lip: a case report and review of literature
}

\author{
Rashmi Metgud $^{1 *}$, Bhardwaj Tina Neelesh $^{2}$, Ranjitha $^{3}$, Saurabh Goel ${ }^{4}$, Smitha Naik $^{5}$, Anirrudh Tak $^{6}$ \\ ${ }^{1}$ Prof. \& HOD, Oral \& Maxillofacial Pathology and Microbiology, Pacific Dental College \& Hospital, Udaipur \\ ${ }^{2}$ Post Graduate Student, Oral \& Maxillofacial Pathology and Microbiology, Pacific Dental College \& Hospital, Udaipur \\ ${ }^{3}$ Senior Lecturer, Oral \& Maxillofacial Surgery, Pacific Dental College \& Hospital, Udaipur \\ ${ }^{4}$ Reader, Oral Medicine \& Radiology, Pacific Dental College \& Hospital, Udaipur \\ ${ }^{5}$ Reader, Oral \& Maxillofacial Pathology and Microbiology, Pacific Dental College \& Hospital, Udaipur \\ ${ }^{6}$ Senior Lecturer, Oral \& Maxillofacial Pathology and Microbiology, Pacific Dental College \& Hospital, Udaipur \\ *Corresponding author E-mail:rashmi_metgud@rediffmail.com
}

\begin{abstract}
Pleomorphic Adenoma is a benign epithelial tumor arising from the salivary gland. It is a benign mixed tumor composed of epithelial and myoepithelial cells arranged with various morphological patterns, demarcated from surrounding tissues by fibrous capsule. In this article, we report an unusual case of 30 year, male with a single, nodular swelling evident in the upper lip which was discovered during a routine dental procedure. Fine Needle Aspiration Cytology (FNAC) was planned for the patient, where a of cytological diagnosis of Pleomorphic Adenoma was rendered, which was confirmed by biopsy. Hence, this highlights, FNAC makes up an important diagnosing tool as the first line of investigation to distinguish between benign and malignant salivary gland neoplasms.
\end{abstract}

Keywords: Salivary Gland Neoplasm; Pleomorphic Adenoma; Upper Lip; FNAC; Histopathological Findings

\section{Introduction}

Salivary gland tumors (SGT) are uncommon and account for $1 \%$ of all head and neck neoplasms.(Jagadishkumar et al. 2014) Pleomorphic adenoma (PA) is the most common benign salivary gland tumor that exhibits a wide cytomorphologic and architectural diversity. It is also known as "Mixed tumor, salivary gland type". The annual incidence is approximately $2-3.5$ cases per 100,000 population.(Jaiswal et al. 2014)

World Health Organization in 1972 defined it as a circumscribed tumor characterized by its pleomorphic or mixed appearance clearly recognizable epithelial tissue being intermingled with tissue of Mucoid, Myxoid and Chondroid appearance.(Verma et al. 2014)

Among all salivary gland tumors, PA is the most frequently encountered lesion $(50 \%)$. It account for $53-77 \%$ of parotid tumors, $44-68 \%$ of submandibular tumors, $6.4 \%$ occur in minor salivary glands. Among minor salivary glands, palate is considered as the most common intraoral site, followed by upper lip and cheek. They are more common in adult females from the 3rd to 5th decades where male : female ratio- 1:1.4, 1:1.7.(Verma et al. 2014) The etiology of PA is unknown. It is epithelial in origin, and clonal chromosome abnormalities with aberrations involving 8q12 and $12 \mathrm{q} 15$ have been described.(Shrestha et al. 2010) The putative pleomorphic adenoma gene (PLAG1) has been mapped to chromosome 8q12.(Reddy et al. 2012)

Histologically, PA consists of cells with epithelial and mesencymal differentiation. The treatment of choice for PA is surgical removal with safety margins, to prevent the recurrence. Recurrence rate of $5-30 \%$ has been found for PA, so a periodic follow- up is must, due to the important relapse potential and aggressive nature of these lesions.(Verma et al. 2014)

\section{Case report}

A 30 years old male reported to the Outpatient Department of Pacific Dental College and Hospital, Udaipur with the chief complaint of dirty teeth and pain in lower right and left front region since 5-6 months (Fig. 1) which was chronic, mild, dull aching, intermittent spontaneous along with burning sensation on cheeks on eating spicy food. During a dental procedure a swelling was accidentally discovered. On inquiry for the same the patient informed that he had noticed the swelling 5 months back. No relevant family or dental history. It was patient's first dental visit. Patient was tobacco smoker and chewer since 5 years. On general physical examination, patient was moderately nourished and built with other vital signs under normal range.

On extra oral examination, no abnormalities were detected. On intraoral examination, buccal mucosa revealed white reticular pattern evident bilaterally suggestive of lichen planus. Pus discharge in gingival sulcus irt 42 suggestive of periodontal abscess. Generalized bleeding on probing on gingiva. On inspection, there was a single, nodular swelling evident irt 12, 13, 14 region labial mucosa, overlying mucosa appeared to be normal which was confirmed on palpation with $1.0 \times 1.0 \mathrm{~cm}$ in size, freely movable and non-tender mass was present. (Fig. 2) All hematological investigations were under normal range. On radiographic evaluation, Orthopantomogram and Intraoral Periapical Radiograph revealed no gross pathology or calcification.

After all the investigations done a provisional diagnosis of lipoma or sebaceous cyst was given. FNAC was performed, a small 
amount of clear fluid was aspirated with cheesy material in fluid and slides were prepared, fixed and stained with PAP and Hematoxylin and Eosin. On cytological evaluation, individual and clusters of epithelial cells with oval, round and few spindle shaped nucleus were evident in the background of connective tissue component of fibers. Cytological diagnosis of Pleomorphic Adenoma was rendered. Surgical excision of the lesion was carried out. (Fig. 3)

Macroscopically: We received 1 bit of soft tissue, creamish white in color with glistening superior surface. Cut surface revealed rubbery, fleshy, mucoid material with homogenous white color in the entire part of the lesion suggestive of a solid mass rather than a cystic mass. (Fig. 4)

On histopathological evaluation, scanner view revealed encapsulated tumor mass with highly cellular areas. Low power view revealed epithelial component formed by ducts and small cyst like spaces that contained eosinophilic coagulum and arranged in small cellular nests, sheets of cells and anastomosing cords. Myoepithelial cells with variable morphology- spindle, plasmacytoid and round cells were also evident. Mesenchymal component was composed of fibrous and myxoid areas with endothelial lined blood capillaries of varying size and shape with RBCs. Capsule revealed thick collagen fibers in association with normal mucous salivary acini. High power view confirmed the above mentioned findings of encapsulated tumor mass, glandular epithelium and mesencymal components.

Hence after, clinicocytopathological correlation, a final diagnosis of pleomorphic adenoma of the minor salivary gland was rendered. After excision no recurrence has been reported.

\section{Discussion}

The minor salivary glands are widely dispersed in the upper respiratory tract, including the lip, palate, pharynx, larynx and nasopharynx. Maximum densities of glands are in the palate.(Jagadishkumar et al., 2014) Tumors originating in the minor salivary glands are uncommon neoplasms.(Verma et al. 2014) PA are the most common benign salivary gland neoplasms.(U et al. 2010) This tumor has been referred to by a great variety of names through the years- Enclavoma, Branchioma, Endothelioma, Enchondroma, but the term Pleomorphic Adenoma suggested by WILLS characterizes closely the unusual histological pattern of the lesion.(Rajendra) The tumors are named Pleomorphic Adenoma because of the epithelial and connective tissue components in varying degrees.(Jagadishkumar et al. 2014)

Regardless of the site of origin, the PA typically appears as a painless, slowly growing, firm mass. The patient may be aware of the lesion for many months or years before seeking a diagnosis as it happened in our presented case.(Neville and Allen) Because of tightly bound nature of the hard palate mucosa, it appears to be fixed. While in cases of lips and buccal mucosa, it is freely movable.(Sharma et al. 2011)

Kroll and Hick reviewed 4042 cases of pleomorphic adenomas of the salivary glands. Of these, 445 originated in the minor salivary glands, only $16.9 \%$ were located in the upper lip and $2.9 \%$ in lower lip. Pleomorphic adenoma in the upper lip exceeds that of the lower lip by the ratio of $6: 1$. The reason for this difference has been thought to be due to the differences in embryonic development between the upper and lower lips. Pleomorphic adenoma arising from minor salivary glands of the lips tends to occur at an earlier age than it does at other sites.(Shrestha et al. 2010)

Pleomorphic adenoma arising from minor salivary glands of the lips tends to occur at an earlier age than it does at other sites. Bernier found that the peak incidence of pleomorphic adenoma of the lips was in the third and fourth decades, with an average age of 33.2 years. There is a propensity for benign tumor to occur in the upper lip, whereas malignant lesions to predominate in the lower lip. Owens and Calcaterra found $90 \%$ of the upper lip tumors to be benign in reports in the literature.(Shrestha et al. 2010)
Fine needle aspiration cytology is a popular method for diagnostic evaluation of salivary gland masses due to their superficial nature and easy accessibility for the procedure. It is a major diagnostic to relevance in head and neck lesions, including salivary glands. In this region, FNAC is easy to perform and achieve excellent patient compliance, being minimally procedure.(Junu \& Kunja 2015)

\section{Criteria for cytological diagnosis}

- Fibrillary chondromyxoid ground substance.

- Variable cellularity, mainly myoepithelial cells, single and in poorly cohesive clusters and sheets.

- Rounded, ovoid, plasmacytoid or spindle cells with abundant well defined cytoplasm.

- Regular ovoid nuceli with bland finely granular nuclear chromatin.

- Spindle shaped myoepithelial cells embedded in stromal matrix.

- Sometimes metaplastic cells (oncocytic, sebaceous, squamous).(Svante et al. 2008)

Histologically, it is typically well circumscribed encapsulated tumor but lack of complete encapsulation is more common for minor salivary gland tumors. Histologically, the tumor mainly comprises of epithelial, myoepithelial cells and mesenchyme like components. Epithelial component shows wide variety of cell types including cuboidal, basaloid, squamous, spindle, plasmacytoid and clear cells unlike the presented case where capsule was present. Epithelium usually forms sheets or duct like structures. Mesenchyme like components is mucoid / Myxoid, cartilaginous or hyalinized. Bone may form within the cartilage or formed directly by osseous metaplasia of the stroma. Myoepithelial cells are the main component of PA. They appear as angular, spindle, or plasmacytoid / hyaline cells and are also responsible for mesenchyme like changes.(Reddy et al. 2012) Dardick has described the neoplastic myoepithelial cells in PA as "swarm of bees" enclosing ductal structures.(Irving)

Foote and Frazell first submitted such histopathological classification of PA in 1954. Seifert et al. proposed similar findings in 1976. According to these authors,

- Type I comprised of principally myxoid variant,

- Type II comprised of Myxoid and cellular variant,

- Type III comprised of predominantly cellular variant and

- Type IV comprised of an extremely cellular variant.

PA of the major salivary glands generally belong to the type I or II categories while the minor salivary gland tumors are more cellular in nature.(Satpathy et al. 2014) Our case was type III .

In most instances, the diagnosis of pleomorphic adenoma is made through a straightforward microscopic identification. However, immunohistochemistry (IHC) may be supportive and helpful in delineating the different cell types and components, as well as in differentiating pleomorphic adenoma from other tumors.(Jaiswal et al. 2014) Immunohistochemically, the ductal cells express CK 3,6,10,11,13,16 and myoepithelial cells are positive for CK 13, 16 AMND 14. The neoplastic myoepithelial cells are also positive for vimentin, S 100 protein, alpha smooth muscle actin, GFAP, calponin, CD 10 and muscle specific actin.(Reddy et al. 2012)

Treatment of pleomorphic adenomas is a complete surgical excision with a surrounding margin of normal tissue, i.e., superficial/total parotidectomy with facial nerve preservation, submandibular gland excision or wide local excision for a minor salivary gland. Simple enucleation of these tumors is what is believed to have led to high local recurrence rates in the past and should be avoided.(Jaiswal et al. 2014) Rupture of the capsule, inadequate removal or enucleation, pseudopodia, capsular penetration or tumor spillage in the wound are also believed to increase the risk of recurrence, so meticulous dissection is paramount.(Jaiswal et al. 2014, Pati et al. 2011) The risk of recurrence is lower in minor salivary gland tumors.(Reddy et al. 2012) In our case, wide local excision along was done and no recurrence has been reported yet. 


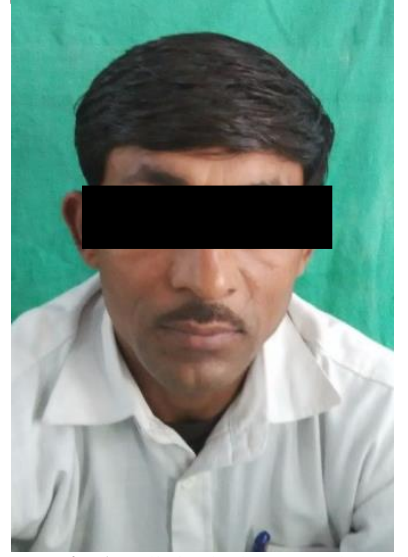

Fig.1: Clinical Photograph

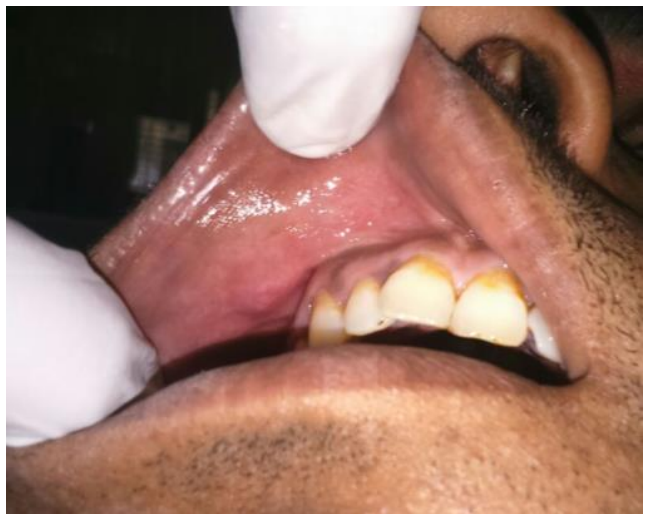

Fig. 2: small nodular swelling in the right side of the upper lip

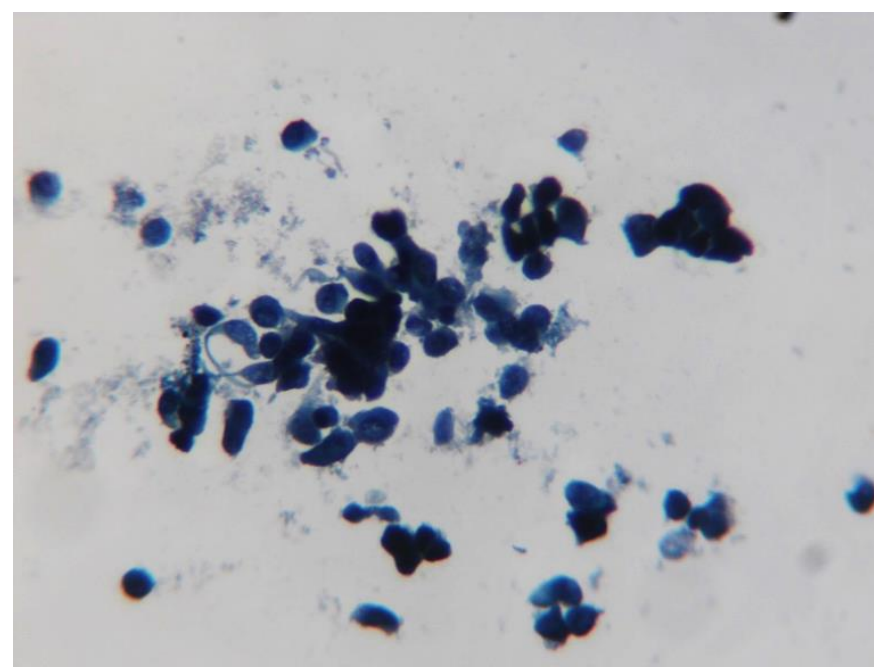

Fig. 3: Cytological evaluation
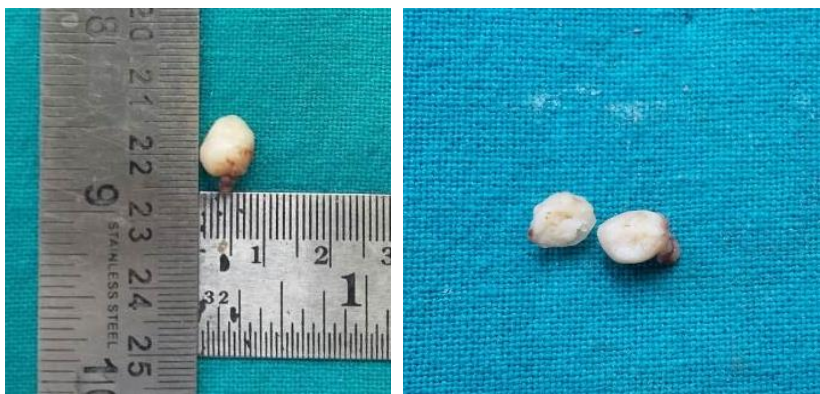

Fig. 4: Macroscopical Evaluation cut surface revealing white homogenous material in the entire part of the specimen.

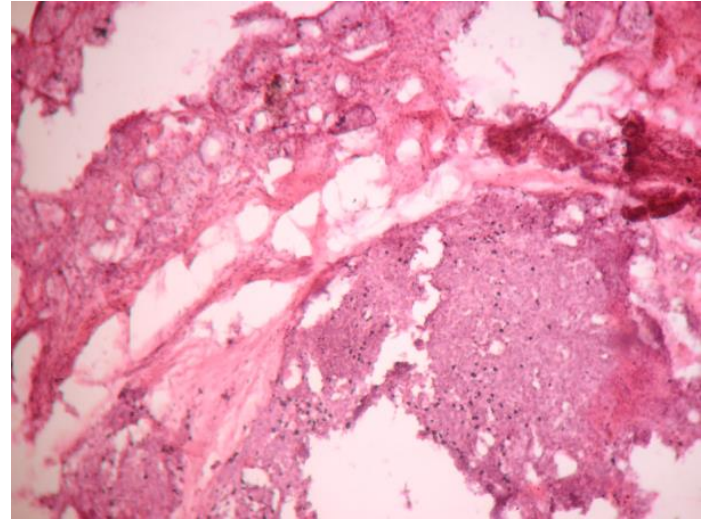

Fig. 5: Histopathological evalution

\section{Conclusion}

Salivary gland tumors are relatively uncommon but their multifaceted clinical presentation; varied morphologic configuration and relatively unpredictable prognosis continue to attract significant medical interest. This case report alerts the clinicians, as these are intraoral swellings which are usually asymptomatic, it may be discovered on routine oral examination. FNAC is a simple, quick inexpensive and minimally invasive technique, can be recommended as a first line of investigation to distinguish between benign and malignant salivary gland neoplasm. However, histopathology is the gold standard for final diagnosis. FNAC has proved to be an aid in diagnosing the present case.

\section{References}

[1] Irving, D. Color Atlas / Text of Salivary Galand Tumor Pathology.

[2] Jagadishkumar, K., Anilkumar, M. G., Kumar, H. C. K. \& Maggad, R (2014) Pleomorphic adenoma of the cheek in a child: A case report. Dental research journal, 11, 522 .

[3] Jaiswal, A. A., Garg, A. K. \& Membally, R (2014) Pleomorphic adenoma of parotid gland with extensive bone formation-A rare case report. Egyptian Journal of Ear, Nose, Throat and Allied Sciences, 15, 139-142. http://dx.doi.org/10.1016/j.ejenta.2013.12.005.

[4] Junu, D. \& Kunja, L., Talukdar (2015) Salivary gland neoplasms: A clinicopathological study of 84 cases. International Archives of Integrated Medicine, 2.

[5] Neville, D. \& Allen, B. Oral and Maxillofacial Pathology, Elsevier.

[6] Pati, A. R., Giraddi, G. B. \& Singh, C (2011) Beningn pleomorphic adenoma of minor salivary gland of palate. Journal of Dentistry and Oral Hygiene, 3, 82-8.

[7] Rajendra, S. Shafer's Textbook of Oral Pathology, Elsevier.

[8] Reddy, M., Mahajan, B., Chandwam, R., Kulkarni, M. \& Desai, R. S. (2012) A Case of Pleomorphic Adenoma of Upper Lip. Indian Journal of Stomatology, 3.

[9] Satpathy, Y., Spadigam, A. E., Dhupar, A. \& Syed, S (2014) Epithelial and stromal patterns of pleomorphic adenoma of minor salivary glands: A histopathological and histochemical study. Journal of oral and maxillofacial pathology: JOMFP, 18, 379. http://dx.doi.org/10.4103/0973-029X.151319.

[10] Sharma, Y., Maria, A. \& Chhabria, A (2011) Pleomorphic adenoma of the palate. National journal of maxillofacial surgery, 2, 169. http://dx.doi.org/10.4103/0975-5950.94473.

[11] Shrestha, A., Reddy, N. \& Ganguly, S (2010) Pleomorphic adenoma of the upper lip: A case report. Journal of College of Medical Sciences-Nepal 6 ,

51-53. http://dx.doi.org/10.3126/jcmsn.v6i1.3603.

[12] Svante, R., Orell, Gregory, F., Sterrett \& Darrel, W (2008) Fine Needle Aspiration Cytology, Elsevier.

[13] U, G., Bl, S., Prof, B., Sinha \& Prof, D., Baskota (2010) Pleomorphic Adenoma of Salivary Gland: an experience at TUTH. Nepalese Journal of ENT Head and Neck Surgery, 1.

[14] Verma, P., Sachdeva, S. K., Verma, K. G. \& Sachdeva, K (2014) Pleomorphic adenoma of cheek: A rare case report and review of literature. Indian Journal of Dental Research, 25, 122.

[15] http://dx.doi.org/10.4103/0970-9290.131166. 